\section{1}

2

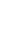

\title{
Title: Intestinal infection results in impaired lung innate immunity to
}

\section{secondary respiratory infection.}

\section{Subtitle: Lung immunity after gut infection.}

Shubhanshi Trivedi, ${ }^{1}$ Allie H. Grossmann, $, 3,5$ Owen Jensen, ${ }^{1}$ Mark J. Cody, ${ }^{4}$ Taylor A. Wahlig, ${ }^{1}$ Paula Hayakawa Serpa, ${ }^{8,9}$ Charles Langelier,${ }^{8,9}$ Kristi J. Warren, ${ }^{2}$ Christian C. Yost, ${ }^{4,6}$ and Daniel T. Leung ${ }^{1,7}$

${ }^{1}$ Division of Infectious Disease, ${ }^{2}$ Division of Pulmonary Medicine, Department of Internal Medicine; ${ }^{3}$ Huntsman Cancer Institute; ${ }^{4}$ Division of Neonatology, Department of Pediatrics; ${ }^{5}$ Division of Anatomic Pathology, ${ }^{6}$ Molecular Medicine Program; ${ }^{7}$ Division of Microbiology and Immunology, Department of Pathology; University of Utah, Salt Lake City, UT, USA; ${ }^{8}$ Chan Zuckerberg Biohub, San Francisco, California, USA; ${ }^{9}$ Division of Infectious Diseases, Department of Medicine, University of California-San Francisco, San Francisco, California, USA.

(1)

Article summary: We show, in a mouse model, that prior intestinal infection with Salmonella Typhimurium leads to increased susceptibility to respiratory Klebsiella pneumoniae infection, which is associated with altered neutrophil responses.

\section{Corresponding author:}

Daniel T Leung, M.D. 
bioRxiv preprint doi: https://doi.org/10.1101/2020.08.03.235457; this version posted March 11, 2021. The copyright holder for this preprint (which was not certified by peer review) is the author/funder. All rights reserved. No reuse allowed without permission.

21 Address: 26 North Medical Drive, Salt Lake City, UT 84132, USA

22 Telephone: 801-581-8804

23 Fax: 801-585-3377

24 Email: daniel.leung@utah.edu

25 


\section{Abstract}

\section{Background}

28 Pneumonia and diarrhea are among the leading causes of death worldwide, and

29 epidemiological studies have demonstrated that diarrhea is associated with an

30 increased risk of subsequent pneumonia. Our aim was to determine the impact of

31 intestinal infection on innate immune responses in the lung.

\section{Methods}

33 Using a mouse model of intestinal infection by Salmonella enterica serovar

34 Typhimurium (S. Typhimurium (ST)), we investigated how infection in the gut

35 compartment can modulate immunity in the lungs and impact susceptibility to bacterial

36 (Klebsiella pneumoniae $(K P))$ challenge.

\section{Results}

38 We found alterations in frequencies of innate immune cells in lungs of intestinally-

39 infected mice compared to uninfected mice. On subsequent challenge with $K$.

40 pneumoniae we found that mice with prior intestinal infection have higher lung bacterial

41 burden and inflammation, increased neutrophil margination, and neutrophil extracellular

42 traps (NETs), but lower overall numbers of neutrophils, compared to mice without prior

43 intestinal infection. Total numbers of dendritic cells, innate-like T cells, and natural killer

44 cells were not different between mice with and without prior intestinal infection. 
46 Together, these results suggest that intestinal infection impacts lung innate immune

47 responses, most notably neutrophil characteristics, potentially resulting in increased

48 susceptibility to secondary pneumonia.

49

50 Keywords: Diarrhea, Pneumonia, Innate immunity, Neutrophils, NETs

51 Abstract: 193 words

52 Main text: 3473 words (not including citation numbers) 


\section{Background}

54 Diarrhea and pneumonia are among the leading causes of death worldwide. In children

55 alone, these diseases combine to kill $\sim 1.4$ million each year, with the majority of these

56 deaths occurring in lower and middle-income countries [1]. Epidemiological studies

57 have shown that children are at an increased risk of pneumonia following a diarrheal

58 episode [2, 3]. However, the immunological mechanisms behind an increased susceptibility to such secondary respiratory infections are not well understood.

Although the gastrointestinal and respiratory tracts have different environments and functions, there is emerging data showing cross-talk between these two mucosal sites in chronic inflammatory diseases such as inflammatory bowel disease (IBD) and asthma [4]. Additionally, there is emerging evidence that the intestinal microbiota plays a role in host defense against bacterial pneumonia [5]. The gastrointestinal and respiratory tracts share the same embryonic origin and have common components of the mucosal immune system such as an epithelial barrier, submucosal lymphoid tissue, the production of IgA and defensins, and the presence of innate lymphocytes and dendritic cells [6]. Notably, several innate-like leukocytes, such as Mucosal Associated Invariant T (MAIT) cells, invariant Natural Killer T (iNKT) cells, gamma delta T ( $ү \delta$ T) cells, dendritic cells (DC) and neutrophils, have the capacity to circulate between tissues, and

71 play important roles in both respiratory and intestinal tract immunity [7, 8].

72 How intestinal infection impacts immunity in the lung is not known. In this study, we

73 examine the impact of intestinal infection on the immune response in the lungs of mice

74 using an established model of intestinal infections. We found that mice infected with $S$. 
75 Typhimurium (ST) have increased susceptibility to respiratory Klebsiella pneumoniae

$76(K P)$ infection compared to mice without prior intestinal infection. Prior intestinal

77 infection modulated effector cells of innate immunity in the lung, contributing to

78 respiratory immune dysregulation and a higher $K P$ bacterial burden.

\section{Methods}

80

81

82

\section{Mice and inoculations}

Six to eight week old female C57BL/6J wild type mice were obtained from Jackson Laboratories. All animals were maintained and experiments were performed in accordance with University of Utah and Institutional Animal Care and Use Committee approved guidelines (protocol 17-01011). The animals were kept at a constant temperature $\left(25^{\circ} \mathrm{C}\right)$ with unlimited access to pellet diet and water in a room with a $12 \mathrm{~h}$ light/dark cycle. All animals were monitored daily and infected animals were scored for the signs of clinical illness severity [9]. Animals were ethically euthanized using $\mathrm{CO}_{2}$.

For the experiments, all mice were pretreated with streptomycin as described previously [10]. Briefly, water and food were withdrawn 4 hours before oral gavage treatment with $7.5 \mathrm{mg}$ of streptomycin in $100 \mu \mathrm{lHBSS}$. Afterward, animals were supplied with water and food ad libitum. At $20 \mathrm{~h}$ after streptomycin treatment, water and food were withdrawn again for 4 hours before mice were gavaged with $10^{4} \mathrm{CFU}$ of ST (100 $\mu \mathrm{l}$ suspension in PBS) or treated with sterile PBS (control). Thereafter, drinking water and food ad libitum was offered immediately. Six days post infection, mice were euthanized and lungs removed for analysis. 
98 For KP challenge experiments, six days post $S T$ infection, isoflurane anesthetized mice

99 were inoculated intranasally with high inoculum 10 ${ }^{10} \mathrm{CFU} K P$ in a $50 \mu$ l volume.

100 Inoculated mice were euthanized at $18 \mathrm{~h}$ for bacterial load enumeration and immune

101 assessment (details of bacterial strains and dosing justifications in Supplementary

102 Methods).

103

104

\section{Lung Histology}

105

After sacrifice, mouse lungs were infused with $10 \%$ neutral buffered formalin via the

trachea, fixed in formalin overnight, dehydrated in $70 \%$ ethyl alcohol and embedded in

paraffin. $4 \mu \mathrm{m}$ sections were stained with haematoxylin and eosin and analysed by a

board certified anatomic pathologist (A.H.G.). Samples were blinded prior to

histopathologic analysis.

\section{Lung Neutrophil Extracellular Trap Assessment}

112 Paraffin imbedded mouse lung were cut to $8 \mu \mathrm{m}$ thickness on a microtome. Sections were deparaffinized and rehydrated using xylene and decreasing ethanol concentration washes. Heat induced epitope antigen retrieval of lung sections were processed in a

1152100 Retriever Thermal Processor (Electron Microscopy Sciences) containing Citrate

116 buffer $\mathrm{pH} 6.0$ solution. Sections were incubated for 10 minutes with $0.1 \%$ Triton-X-100

117 and blocked with 10\% Donkey Serum for 1 hour at RT. Antibodies for citrullinated

118 Histone H3 (Abcam) and myeloperoxidase (MPO; R\&D Systems) were incubated at

$1191: 100$ dilution in $10 \%$ donkey serum overnight at $4^{\circ} \mathrm{C}$. After washing sections with PBS, 
121 respectively, along with DAPI nuclear stain were incubated on sections for 90 minutes

122 at $4^{\circ} \mathrm{C}$. Sections were washed and coverslips were adhered with aqueous mounting

123 medium (Dako, S3025). Images were acquired on an Olympus FV3000 Confocal Laser

124 Scanning Microscope. FluoView software (Olympus) and ImageJ Fiji (NIH) were used

125 for image processing and analysis. We quantified NET formation on the images using a

126 standardized grid system [11]. Briefly, we placed a standardized grid on randomly

127 selected high-power field images ( $n=5$ field images/sample). The number of times that

128 any NET crossed a grid line was tallied.

Lung mononuclear cell isolation

131 For lung digestion and preparation of single cell suspensions, lungs were perfused 132 using $5 \mathrm{~mL}$ PBS, aseptically harvested from euthanized mice and kept in RPMI with $13310 \%$ Fetal Bovine Serum (FBS). Lungs were dissociated using the mouse Lung

134 Dissociation Kit (Miltenyi Biotec) and the gentleMACS Dissociator (Miltenyi Biotec).

135 Cells were then passed through a $70 \mu \mathrm{m}$ cell strainer and washed. Red blood cells were 136 lysed with red blood cell lysis buffer. Lung mononuclear cells were then washed twice.

138 Bacterial Load quantification

$139 K P$ bacterial load was determined by plating ten-fold serial dilutions of the lung

140 homogenates onto MacConkey agar plates (Sigma-Aldrich). The plates were incubated 141 at $37^{\circ} \mathrm{C}$ overnight before bacterial CFUs were determined by colony counts. 
144 Lungs homogenates were filtered on $70 \mu \mathrm{m}$ cell strainers and centrifuged at $300 \times \mathrm{g}$ for

$1455 \mathrm{~min}$. Supernatants were stored at $-80^{\circ} \mathrm{C}$ for cytokine content analysis. Lung cytokine

146 levels were assessed from the supernatant samples via LEGENDplex kit (mouse

147 inflammation panel 13-plex; BioLegend) per manufacturer's instructions. Cytokine levels

148 were acquired using a FACSCanto II flow cytometer (BD Biosciences), and analyses

149 were performed using LEGENDplex data analysis software (BioLegend).

150

151 Tetramer and antibody surface-staining of lung single cell suspensions

152 From each animal, 1-2 million cell aliquots were prepared and stained with the fixable

153 viability dye eFluor ${ }^{\mathrm{TM}} 780$ (eBioscience) for $15 \mathrm{~min}$ at room temperature (RT) to exclude

154 dead cells, and washed with PBS $+2 \%$ FBS and incubated with anti-mouse

155 CD16/CD32 Fc Block antibody (BD), for 20 min at $4{ }^{\circ} \mathrm{C}$. Cells were then stained for 30

156 min with fluorescence-conjugated antibodies at either RT or $4^{\circ} \mathrm{C}$ (detailed in

157 Supplementary Methods). A total of $10^{6}$ gated events per sample were collected using

158 the BD Fortessa flow cytometer, and results analyzed using FlowJo 10.4.2 software.

\section{Statistical analysis}

161 GraphPad Prism 8 software was used for statistical analysis. The Mann-Whitney $U$ test

162 was used for comparison between uninfected and ST infected groups and between

163 mice with and without prior intestinal infection. Results were presented as mean \pm standard deviation, and $p<0.05$ was considered statistically significant. 


\section{Results}

\section{Mice with prior ST intestinal infection have increased lung bacterial load,} sickness score, and susceptibility to respiratory $K P$ infection.

To test whether prior intestinal infection increases the susceptibility to respiratory $K P$ infection, we evaluated survival, body weight loss, sickness score, and bacterial burden in lungs post KP challenge (Fig 1A). Compared to ST infected mice, which had a survival rate of $90-100 \%$, and K. pneumonia infected mice, which had a survival of 60 $80 \%$, mice with prior ST intestinal infection had a survival rate of $0-30 \%$ at 120 hours

173 post $K P$ infection (Fig 1B). Interestingly, although there were no statistically significant differences in body weight loss between mice with or without ST infection with KP challenge (Fig S1), mice with prior intestinal infection had a higher sickness score (Fig 1C). This sickness scoring system included hunched posture, ruffled fur, decreased movement and altered respiratory rates and quality of breaths (Fig 1C). Of note, mice with only ST intestinal infection showed no weight loss and had reduced sickness scores. When bacterial burden was evaluated at 18 and 33 hours post $K P$ infection, we found significantly higher lung bacterial burden in mice with prior intestinal infection compared to mice without prior intestinal infection (Fig 1D). Mice with prior intestinal infection also showed significantly higher lung bacterial burden at 33 hours compared to 18 hours (Fig 1D). We could not isolate any ST from lung homogenates of mice with intestinal infection. In addition, in a subset of animals, we performed metagenomic next generation sequencing (Supplementary Methods) and observed the presence of ST in 
2. Mice with prior ST intestinal infection have increased lung inflammation from subsequent respiratory $K P$ infection.

We next examined the degree of lung inflammation by histological analysis of tissue sections from all four groups of mice. Histopathological analysis revealed mixed interstitial inflammatory consolidations in mice with $K P$ respiratory infection and increased microabcess formation with pyknotic neutrophils in mice co-infected with ST and $K P$ (Fig 2A and supplementary table 2). Uninfected mice and mice with intestinal infection showed normal alveolar and interstitial lung histology (Fig 2A). Upon challenge with $K P$ respiratory infection, mice with prior $S T$ intestinal infection also showed marked intravascular clustering of polymorphonuclear neutrophils (PMNs), with increased margination, necrotic cluster formation and extravasation (Fig 2B and Fig S2). Intravascular neutrophil clustering was noticeably absent in the other treatment groups. Furthermore, lung sections from mice with intestinal infection, and mice with both intestinal and respiratory infection, showed scattered microthrombi in capillary-sized vessels whereas mice with $K P$ respiratory infection (with no prior intestinal infection) showed no microthrombi formation (Fig S3).

\section{$K P$ challenge.}

207 The delicate balance between pro- and anti-inflammatory cytokines is crucial in containing pathogens and maintaining tissue repair and homeostasis in the lung [11]. neutrophils to the lung tissue $[12,13]$. We investigated whether intestinal infection 
211 affected cytokine production in lung homogenates, and whether prior intestinal infection

212 affected this cytokine response to intranasal $K P$ challenge. Our results indicate that

213 compared with the uninfected control group, ST infected mice had significantly higher

214 lung levels of IFN-y, MCP-1 and IL-1ß (Fig 3 A-C). While Thy1-expressing natural killer

215 (NK) cells [14] and NKp46+ ILC3 [14] cells are commonly thought as the sources of IFN-

$216 \mathrm{Y}$, there is a mounting evidence that neutrophils are a prominent cellular source of IFN-Y

217 during the innate phase of $S T$-induced colitis [15]. It is possible that large numbers of

218 primed neutrophils traffick to lungs after intestinal infection, contribute to cytokine

219 production and increase the potential for neutrophil-mediated pathology or NET

220 formation upon secondary infection [8]. Furthermore, following KP challenge, mice with

221 prior intestinal infection had higher levels of IFN-y and lower levels of GM-CSF cytokine

222 production in lung homogenates compared to those without prior intestinal infection (Fig

223 3A and D). Levels of IL-23, IL-1 $\alpha$, TNFa, IL-12p70, IL-10, IL-6, IL-27, IL-17A and IFN- $\beta$

224 were not significantly different between mice with and without prior intestinal infection

225 (Fig S4).

226

227 lungs after $K \boldsymbol{P}$ challenge.

228

229

230

231

232

233

\section{Mice with prior ST intestinal infection have lower numbers of neutrophils in the}

We next examined the impact of intestinal infection on innate cellular responses in the lung, and also its effect on such responses to $K P$ respiratory challenge. We analyzed changes in major innate lung leukocytes (plasmacytoid dendritic cells (pDCs), monocyte-derived dendritic cells (moDCs), CD103+ DCs, neutrophils, alveolar macrophages (AMs) and interstitial macrophages (IMs)) pre- and post-KP challenge in mice infected with ST (gating strategy in Fig S5A). Consistent with previous studies [9], 
234 we observed rapid and robust recruitment of neutrophils to the lungs at 18 hours

235 following $K P$ infection compared to uninfected controls. Interestingly, mice with prior

236 intestinal ST infection had significantly lower frequencies and total number of lung

237 neutrophils following $K P$ challenge compared to mice infected with $K P$ alone (Fig 4A).

238 Furthermore, results indicated that frequencies of pDCs increased and moDCs

239 decreased in the lungs of ST intestinally infected mice compared to uninfected controls

240 (Fig 4B and C). Following intranasal KP challenge, we found a marked increase in

241 frequencies of pDCs and significantly lower frequencies of moDCs in mice with prior ST

242 intestinal infection compared to those without prior intestinal infection (Fig 4B and C).

243 No significant differences were observed in frequencies of CD103+ DCs (Fig 4D)

244 between KP infected mice with and without prior ST intestinal infection. Total numbers

245 of pDCs, moDCs, CD103 ${ }^{+}$DCs, AMs or IMs were not different in mice with prior

246 intestinal infection compared to those without prior ST intestinal infection (Fig 4 and Fig

247 S6). We also found higher numbers of neutrophils, pDCs and IMs and lower numbers of

$248 \mathrm{CD}_{103^{+}} \mathrm{DCs}$ in mice with only ST intestinal infection compared to uninfected mice (Fig 4 249 and Fig S6).

251 We also investigated innate-like T cells including mucosal-associated invariant T (MAIT)

252 cells, invariant natural killer T (iNKT) cells, үס T cells, and natural killer cells (NK) as

253 they are known to play an important role in bacterial infections (gating strategy in Fig

254 S5B) [10]. No differences were observed in percentage frequencies or total number of

255 iNKT cells, MAITs, y $\delta$ T cells or NK cells between mice with and without prior intestinal

256 infection (Fig 5). Furthermore, when complete blood counts were assessed with a 
257 Hemavet analyzer, no significant differences were observed in circulating neutrophils,

258 lymphocytes, monocytes, eosinophils, basophils and platelets between mice with and 259 without prior intestinal infection (Fig S7).

\section{Mice with prior ST intestinal infection demonstrate widespread lung NETosis}

\section{after $K \boldsymbol{P}$ challenge.}

Recent studies have revealed that excessive NET formation plays a role in pathogenby releasing NETs [20]. Moreover, we detected higher levels of IFN- $y$ in mice with prior intestinal infection compared to mice without prior intestinal infection (Fig 3), and IFN-y can promote NET formation by neutrophils [21]. We therefore determined whether prior intestinal ST infection was associated with increased lung NET formation following intranasal KP challenge. We examined citrullinated histones and MPO in lung tissues of

273 each experimental group by immunofluorescence. We found a significantly higher

274 number of NETs $(p=0.0006)$ in mice with prior intestinal ST infection compared to

275 those without prior intestinal infection (Fig $5 \mathrm{E}$ and F), as demonstrated by the presence

276 of extracellular DNA overlaid with citrullinated histone H3 and MPO (Fig 5E). Mock-

277 infected lungs did not show any staining for citrullinated histone $\mathrm{H} 3$ or MPO. We 
bioRxiv preprint doi: https://doi.org/10.1101/2020.08.03.235457; this version posted March 11, 2021. The copyright holder for this preprint (which was not certified by peer review) is the author/funder. All rights reserved. No reuse allowed without permission.

$279 S T$ infection alone, and $K P$ infected alone groups, and there was no significant

280 difference between these groups (Fig 5F). 


\section{Discussion}

282

283

284

285

286

287

288

289

290

291

292

293

294

295

296

297

298

299

300

301

302

We found that bacterial intestinal infection in mice adversely impacts immunity in the lung, increasing susceptibility to secondary respiratory infection. We show that mice with prior intestinal ST infection have higher lung bacterial burden and sickness scores after subsequent $K P$ challenge compared to mice without prior $S T$ infection. This finding was associated with changes in innate cellular responses, most notably those of neutrophils, which were decreased in the parenchyma, clustering in the lung vasculature, and associated with increased NET formation in those with secondary infection. As neutrophils are essential for pulmonary clearance of bacterial infections such as $K P$ [22], it is possible that intestinal infection impairs the recruitment and function of lung neutrophil responses against $K P$, leading to an inability to clear the lung bacterial infection, thereby worsening lung function.

In addition to epidemiological studies showing a higher susceptibility to pulmonary infections after intestinal infection in children [2, 3], and that intestinal diseases are often associated with pulmonary disorders [23], the immunological crosstalk of the lung-gut axis is not well understood. In the context of IBD, it has been proposed that intestinal inflammation and increased cytokine levels create conditions favorable for neutrophil margination onto the lung endothelium [24]. When the lung encounters a secondary insult, neutrophil recruitment, activation and extravasation could mediate lung tissue injury and IBD-induced respiratory diseases [8, 25]. Our knowledge of how intestinal infection impacts the recruitment, extravasation and function of neutrophils in tissues 
303

304

305

306

307

308

309

310

311

312

313

314

315

316

317

outside of the intestine is limited. Studies have shown that GM-CSF plays an important role in neutrophil accumulation [26, 27], and GM-CSF is protective in preclinical models of pneumonia-associated lung injury [28]. We found a significantly lower lung GM-CSF response to $K P$ infection in mice with prior intestinal infection, which may decrease the lung's ability to recruit circulating neutrophils, resulting in an increased susceptibility to infection. In line with this, we also detected increased NET formation and microthrombosis in $K P$ infection in mice with prior intestinal $S T$ infection as compared to mice without prior intestinal infection. It is likely that NETs and NET-associated factors, including histones and granule proteases, mediate vascular and tissue injury and contribute to microthrombosis [11, 29-31]. The activation of NETosis, the regulated cell death process leading to NET formation, also causes changes in neutrophil morphology including cell membrane rupture and neutrophil death [32]. We speculate that prior intestinal infection induces NET formation contributing to lower numbers of viable neutrophils in the lungs. Further studies examining the mechanisms governing neutrophil activation and NET formation in lungs after intestinal infection are warranted.

Nonconventional T lymphocytes including MAIT cells, NKT cells and yס-T cells, have tissue-homing properties, and have been implicated in protection against respiratory bacterial infections [7]. Here, we found no differences in frequencies or number of these cells between the groups tested. Likewise, except for IFN-y we did not find any differences in cytokines that have been implicated in lung defense against bacterial pathogens, including IL-17A [33], TNF-a [34] and IL-10 [35]. Both a protective [36] and detrimental [37] role of IFN-y has been reported in bacterial infections, and we found 
significant increases in lung IFN-y production in mice with intestinal infection. Numerous studies have shown that IFN-y produced by activated $\mathrm{CD}^{+}$and $\mathrm{CD} 8^{+} \mathrm{T}$ cells and $\mathrm{NK}$ cells plays an important role in protective immunity to Salmonella [38, 39]. Although we did not observe differences in NK cell numbers in mice with and without prior intestinal infection, it is possible that activated T cells responding to conserved microbial epitopes are recruited early to the lung after intestinal infection and are a major source of IFN-y production. However, the importance of T cell activation and IFN- $y$ production in our coinfection model is unclear and merits further investigation. Modulation of respiratory DCs during KP infection has been reported before by Hackstein et al. [40]. Although we did not observed differences in total number of DCs, we observed higher frequencies of pDCs in lungs of mice with prior intestinal infection, and this may be related to the role of respiratory pDCs in tissue repair [41]. Alternatively, accumulation of pDCs in lungs post intestinal infection may have involvement in the initiation of inflammation and antigen-specific $T$ cell responses [42]. Moreover, frequencies of moDCs, which may contribute to control of secondary respiratory KP infection, was decreased in mice with prior intestinal infection [43]. Further studies are necessary to address the functional role of pDCs and moDCs during bacterial pneumonia after intestinal infection.

Our study has several limitations. Firstly, we did not account for the effect of the preexisting intestinal microbiota; however, all mice were purchased from the same source, and were cohoused prior to infection. Secondly, we did not evaluate serum levels of IL6, TNF- $\alpha$, IFN-y and VEGF [24] and neutrophil chemokines such as keratinocytederived chemokine $(\mathrm{KC})$, macrophage inflammatory protein $2, \mathrm{CXC}$ receptor 2 and 
CXC ligand 5 [44] which would further our understanding of lung neutrophil trafficking

350 following intestinal inflammation. Thirdly, we have not investigated the role of innate

351 lymphoid cells, which have been shown to be recruited from gut to the lungs in

352 response to inflammation [45]. Fourthly, we have not tested the impact of lower/graded

353 doses of $K P$ challenge in mice with prior intestinal infection. Lastly, we emphasize that

354 our study lacks experiments aimed at determining the mechanisms leading to increased

355 lung pathology following intestinal infection. We hypothesize that in secondary

356 infections, neutrophil response and NET production requires a fine balance:

357 underactivity can lead to risk for increased pathogen replication and invasion, whereas

358 over-activity can result in excessive inflammation and tissue damage. Future

359 experiments examining the effect of neutrophil depletion and the effect of NET inhibitors

360 and NET dismantling agents such as DNase are warranted.

362 In conclusion, the present study demonstrates that infection in the gut adversely

363 impacts immunity in the lung. This report opens up potential avenues for investigating

364 the immunological crosstalk between the lung and gut during enteric infection. While

365 epidemiological studies have demonstrated this lung-gut association, we provide here

366 novel findings that intestinal infection modulates neutrophil and cytokine responses in

367 the lung, resulting in an increased susceptibility to a secondary pneumonia challenge.

368 These data have the potential to inform efforts to prevent and treat respiratory infections

369 in those with intestinal infection or inflammation. 
Notes

371 Acknowledgements. We would like to thank Cole Anderson, Michael Graves,

372 Alexandra Heitkamp and Melanie Prettyman for their technical assistance.

373 Author contributions. S.T. and D.T.L. designed and directed the project. S.T., O.J.

374 and M.J.C. carried out the experiments. D.T.L., A.H.G., K.J.W., C.C.Y. contributed to

375 data analysis. S.T. and D.T.L. wrote the paper. P.H.S. and C.L. conducted

376 metagenomic next generation sequencing. T.W. analyzed metagenomic sequencing

377 data. All authors discussed the results and commented on the manuscript.

378 Financial support. This work was supported in part by grant W81XWH-17-1-0109 from

379 the Department of Defense (to D.T.L.), and by the National Institutes of Health

380 (R01HD093826 to C.C.Y. and R01Al130378 to D.T.L.). This work was supported by the

381 University of Utah Flow Cytometry Facility in addition to the National Cancer Institute

382 through Award Number 5P30CA042014-24, and CMC animal facility.

383 Potential conflicts of interest. C.C.Y. authors a US patent (patent no. 10,232,023 B2)

384 held by the University of Utah for the use of NET-inhibitory peptides for the "treatment of

385 and prophylaxis against inflammatory disorders," for which PEEL Therapeutics, Inc.

386 holds the exclusive license. 
387

388

389

390

391

392

393

394

395

396

397

398

399

400

401

402

403

404

405

406

407

408 409 Experimental Laboratory Mice. Current Protocols in Mouse Biology 2012; 2:145-65.

\section{References}

1. Liu L, Oza S, Hogan D, et al. Global, regional, and national causes of under-5 mortality in 2000-15: an updated systematic analysis with implications for the Sustainable Development Goals. The Lancet 2016; 388:3027-35.

2. Leung DT, Das SK, Faruque ASG, et al. Concurrent Pneumonia in Children Under 5 Years of Age Presenting to a Diarrheal Hospital in Dhaka, Bangladesh. The American Journal of Tropical Medicine and Hygiene 2015; 93:831-5.

3. Schmidt WP, Cairncross S, Barreto ML, Clasen T, Genser B. Recent diarrhoeal illness and risk of lower respiratory infections in children under the age of 5 years. 2009; 38:766-72.

4. Tulic MK, Piche T, Verhasselt V. Lung-gut cross-talk: evidence, mechanisms and implications for the mucosal inflammatory diseases. Clinical \& Experimental Allergy 2016; 46:519-28.

5. Schuijt TJ, Lankelma JM, Scicluna BP, et al. The gut microbiota plays a protective role in the host defence against pneumococcal pneumonia. Gut 2016; 65:575-83.

6. Budden KF, Gellatly SL, Wood DLA, et al. Emerging pathogenic links between microbiota and the gut-lung axis. Nature Reviews Microbiology 2017; 15:55-63.

7. Ivanov S, Paget C, Trottein F. Role of Non-conventional T Lymphocytes in Respiratory Infections: The Case of the Pneumococcus. 2014; 10:e1004300.

8. Mateer SW, Maltby S, Marks E, et al. Potential mechanisms regulating pulmonary pathology in inflammatory bowel disease. 2015; 98:727-37.

9. Burkholder T, Foltz C, Karlsson E, Linton CG, Smith JM. Health Evaluation of 
10. Barthel M, Hapfelmeier S, Quintanilla-Martinez L, et al. Pretreatment of Mice with

411 Streptomycin Provides a Salmonella enterica Serovar Typhimurium Colitis Model That

412 Allows Analysis of Both Pathogen and Host. 2003; 71:2839-58.

413 11. Yost CC, Schwertz H, Cody MJ, et al. Neonatal NET-inhibitory factor and related

414 peptides inhibit neutrophil extracellular trap formation. J Clin Invest 2016; 126:3783-98.

415 12. Yamada M, Gomez JC, Chugh PE, et al. Interferon-y production by neutrophils

416 during bacterial pneumonia in mice. American journal of respiratory and critical care

417 medicine 2011; 183:1391-401.

418 13. Pechous RD. With Friends Like These: The Complex Role of Neutrophils in the

419 Progression of Severe Pneumonia. Front Cell Infect Microbiol 2017; 7:160-.

420 14. Kupz A, Scott TA, Belz GT, et al. Contribution of Thy1+ NK cells to protective IFN-y

421 production during Salmonella typhimurium infections. Proc Natl Acad Sci U S A 2013;

$422 \quad 110: 2252-7$.

423 15. Spees AM, Kingsbury DD, Wangdi T, Xavier MN, Tsolis RM, Bäumler AJ.

424 Neutrophils Are a Source of Gamma Interferon during Acute <span class="named-

425 content genus-species" id="named-content-1">Salmonella enterica</span> Serovar

426 Typhimurium Colitis. Infection and Immunity 2014; 82:1692-7.

427 16. Narasaraju T, Yang E, Samy RP, et al. Excessive neutrophils and neutrophil

428 extracellular traps contribute to acute lung injury of influenza pneumonitis. Am J Pathol

$429 \quad 2011 ; 179: 199-210$.

430 17. Porto BN, Stein RT. Neutrophil Extracellular Traps in Pulmonary Diseases: Too

431 Much of a Good Thing? Frontiers in immunology 2016; 7:311-. 
432 18. Lefrançais E, Mallavia B, Zhuo H, Calfee CS, Looney MR. Maladaptive role of

433 neutrophil extracellular traps in pathogen-induced lung injury. JCI Insight 2018;

$434 \quad 3: e 98178$.

435 19. Pulavendran S, Prasanthi M, Ramachandran A, et al. Production of Neutrophil

436 Extracellular Traps Contributes to the Pathogenesis of Francisella tularemia. Frontiers

437 in Immunology 2020; 11.

438 20. von Brühl ML, Stark K, Steinhart A, et al. Monocytes, neutrophils, and platelets

439 cooperate to initiate and propagate venous thrombosis in mice in vivo. J Exp Med 2012;

$440 \quad 209: 819-35$.

441 21. Bertin F-R, Rys RN, Mathieu C, Laurance S, Lemarié CA, Blostein MD. Natural killer

442 cells induce neutrophil extracellular trap formation in venous thrombosis. Journal of

443 Thrombosis and Haemostasis 2019; 17:403-14.

444 22. Xiong H, Carter RA, Leiner IM, et al. Distinct Contributions of Neutrophils and

445 CCR2+ Monocytes to Pulmonary Clearance of Different Klebsiella pneumoniae Strains.

446 Infection and Immunity 2015; 83:3418-27.

447 23. Kelly MG, Frizelle FA, Thornley PT, Beckert L, Epton M, Lynch AC. Inflammatory

448 bowel disease and the lung: is there a link between surgery and bronchiectasis?

449 International Journal of Colorectal Disease 2006; 21:754-7.

450 24. Scaldaferri F, Vetrano S, Sans M, et al. VEGF-A Links Angiogenesis and

451 Inflammation in Inflammatory Bowel Disease Pathogenesis. 2009; 136:585-95.e5.

452 25. Margraf A, Ley K, Zarbock A. Neutrophil Recruitment: From Model Systems to

453 Tissue-Specific Patterns. Trends in Immunology 2019. 
454

455

456

457

458

459

460

461

462

463

464 465

466

467

468

469

470

471 for bacterial clearance in a murine model of hypersensitivity pneumonitis. Journal of

472 immunology (Baltimore, Md : 1950) 2009; 182:6540-9.

473 34. Skerrett SJ, Martin TR, Chi EY, Peschon JJ, Mohler KM, Wilson CB. Role of the 474 type 1 TNF receptor in lung inflammation after inhalation of endotoxin or Pseudomonas 475 aeruginosa. Am J Physiol 1999; 276:L715-27. 
476

477

478

479

480

481

482

483

484

485

486

487

488

489

490

491

492

493

494

495

496

497

35. Kang MJ, Jang AR, Park JY, et al. IL-10 Protects Mice From the Lung Infection of Acinetobacter baumannii and Contributes to Bacterial Clearance by Regulating STAT3Mediated MARCO Expression in Macrophages. Front Immunol 2020; 11:270.

36. Moore TA, Perry ML, Getsoian AG, Newstead MW, Standiford TJ. Divergent role of gamma interferon in a murine model of pulmonary versus systemic Klebsiella pneumoniae infection. Infection and immunity 2002; 70:6310-8.

37. Schultz MJ, Rijneveld AW, Speelman P, van Deventer SJ, van der Poll T. Endogenous interferon-gamma impairs bacterial clearance from lungs during Pseudomonas aeruginosa pneumonia. Eur Cytokine Netw 2001; 12:39-44.

38. Mittrücker H-W, Köhler A, Kaufmann SHE. Characterization of the Murine TLymphocyte Response to <em >Salmonella enterica</em $>$ Serovar Typhimurium Infection. Infection and Immunity 2002; 70:199-203.

39. Ravindran R, McSorley SJ. Tracking the dynamics of T-cell activation in response to Salmonella infection. Immunology 2005; 114:450-8.

40. Hackstein H, Kranz S, Lippitsch A, et al. Modulation of respiratory dendritic cells during Klebsiella pneumonia infection. Respir Res 2013; 14:91-.

41. Gregorio J, Meller S, Conrad C, et al. Plasmacytoid dendritic cells sense skin injury and promote wound healing through type I interferons. J Exp Med 2010; 207:2921-30.

42. Takagi H, Fukaya T, Eizumi K, et al. Plasmacytoid dendritic cells are crucial for the initiation of inflammation and T cell immunity in vivo. Immunity 2011 ; 35:958-71.

43. Bieber K, Autenrieth SE. Dendritic cell development in infection. Molecular Immunology 2020; 121:111-7. 
498 44. Tateda K, Moore TA, Newstead MW, et al. Chemokine-dependent neutrophil

499 recruitment in a murine model of Legionella pneumonia: potential role of neutrophils as

500 immunoregulatory cells. Infect Immun 2001; 69:2017-24.

501 45. Mjösberg J, Rao A. Lung inflammation originating in the gut. Science 2018; 359:36-

5027.

503 
Figure 1: Mice with prior ST intestinal infection have increased susceptibility to intestinal ST infection and challenged with $K P$ infection $(\mathrm{S}+\mathrm{K})$. Statistical analysis was performed using log-rank (Mantel-Cox) test, $p=0.07$ and log-rank test for significant trend, $p=0.02(C)$ Sickness score plots where data represent cumulative results of two

511 independent experiments ( $n=4-6$ for $S, n=11$ for $K$ and $n=10$ for $S+K$ ) and mean \pm

$512 S D,{ }^{*}$ denotes $p<0.05$. Statistical analysis was performed using Kruskal-Wallis test

513 followed by Dunn's multiple comparison test. (D) KP bacterial load determined in lungs

514 at 18 hours post $K P$ infection in both $\mathrm{K}$ and $\mathrm{S}+\mathrm{K}$ groups. Data represents cumulative

515 results of three independent experiments ( $n=16$ for $K$ and $n=19$ for $S+K)$. Lung

516 bacterial loads also determined at 33 hours in both groups ( $n=4$ for each group, $N=1$

517 experiment). Data represent mean \pm SD and statistical analysis was determined using

518 Mann-Whitney test; * denotes $p<0.05,{ }^{* *}$ denotes $p<0.01$.

520 from subsequent respiratory $K \boldsymbol{P}$ infection, characterized by microabcess, 521 pyknotic neutrophil clusters and margination, compared to those with no prior

522 intestinal infection. (A) Representative images (400x magnification, scale bar $=20$ $523 \mu \mathrm{m})$ of lung sections stained with hematoxylin/eosin, insets shows digital magnification 524 of original image. In uninfected mice and in mice with intestinal infection only (S), black 525 arrows show normal lung parenchyma. Mice with respiratory infection only (K) show 
526 mixed interstitial inflammatory consolidations, and black arrow shows a normal

527 neutrophil. In mice with prior intestinal ST infection and challenged with KP infection

$528(\mathrm{~S}+\mathrm{K})$, microabcess are circled and black arrows highlight clusters of pyknotic

529 neutrophils. (B) Representative images (H \& E, 400x magnification, scale bar $=20 \mu \mathrm{m})$

530 of lung sections of $\mathrm{S}+\mathrm{K}$ infected mice. Lung vessels in $\mathrm{S}+\mathrm{K}$ infected mice showing

531 neutrophil margination (left, black arrows), margination and clustering (middle), necrotic

532 clusters (right, green arrow) and extravasation (right, black arrow). Data represents

533 cumulative results of two independent experiments $(\mathrm{n}=2$ for $\mathrm{UI}, \mathrm{n}=10$ for $\mathrm{S}$ group, $\mathrm{n}=$

5349 for $\mathrm{K}$ group and $\mathrm{n}=8$ for $\mathrm{S}+\mathrm{K}$ group).

536 Figure 3: Mice with prior ST intestinal infection have altered lung cytokine

537 profiles, and altered cytokine responses to respiratory $K \boldsymbol{P}$ infection, compared to

538 those with no prior intestinal infection. Levels of lung inflammatory cytokines (A)

539 IFN-y (minimum detection limit $(\mathrm{MDL})=2.44 \mathrm{pg} / \mathrm{ml})),(\mathrm{B}) \mathrm{MCP}-1(\mathrm{MDL}=2.44 \mathrm{pg} / \mathrm{ml})$,

540 (C) IL-1 $(\mathrm{MDL}=2.44 \mathrm{pg} / \mathrm{ml})$ and $(\mathrm{D}) \mathrm{GM}-\mathrm{CSF}(\mathrm{MDL}=2.44 \mathrm{pg} / \mathrm{ml})$ were assessed 18

541 hours post KP challenge via bead-based LEGENDplex mouse inflammation panel 13-

542 plex assay. Data represents one experiment for uninfected mice $(n=6)$ and $S T$ infected

543 mice $(n=6)$ and cumulative results of three independent experiments for $K(n=18)$ and

$544 S+K$ group $(n=19)$. Error bars represents mean \pm SD and significance was determined

545 by Mann-Whitney tests.

547 Figure 4: Mice with prior ST intestinal infection have altered frequencies of innate 548 cell types in the lung, and altered lung innate cellular responses to respiratory $K \boldsymbol{P}$ 
549 infection. Percentage frequencies, total numbers and representative FACS plots of (A)

550 neutrophils, (B) lung plasmacytoid dendritic cells (pDCs), (C) monocytic dendritic cells

551 (moDCs), (D) CD103+ DCs are shown in uninfected mice (UI), ST infected mice (S), KP

552 infected mice $(\mathrm{K})$ and both $S T$ and $K P$ infected mice $(\mathrm{S}+\mathrm{K})$. Data represents cumulative

553 results of five independent experiments $(n=22$ for $\mathrm{UI}, \mathrm{n}=24$ for $\mathrm{S}, \mathrm{n}=25$ for $\mathrm{K}$ and $\mathrm{n}=$

55422 for $S+K)$. Error bars represents mean \pm SD and statistical significance difference

555 between UI and S or between $\mathrm{K}$ and S+K was determined using Mann-Whitney tests.

556 Figure 5: Mice with prior ST intestinal infection have no differences in iNKT, MAIT,

557 TCRYס and NK cells and have increased NET formation in lungs from subsequent

558 respiratory $K P$ infection, compared to those with no prior intestinal infection.

559 Percentage frequencies, total numbers and representative FACS plots of $(A)$ iNKT cells,

560 (B) MAIT cells and (C) TCR Yס cells and (D) NK cells are shown in uninfected mice

561 (UI), ST infected mice (S), KP infected mice (K) and both $S T$ and $K P$ infected mice

$562(\mathrm{~S}+\mathrm{K})$. Data represents cumulative results of five independent experiments $(\mathrm{n}=22$ for

563 UI, $n=24$ for $S, n=25$ for $K$ and $n=22$ for $S+K)$. Error bars represents mean \pm SD and

564 statistically significance differences between $\mathrm{UI}$ and $\mathrm{S}$ or between $\mathrm{K}$ and $\mathrm{S}+\mathrm{K}$ were

565 determined by using Mann-Whitney tests. (E) Using confocal microscopy, NET

566 formation was assessed in all four groups and images were taken at 60X magnification.

567 Blue fluorescence = nuclear DNA; green fluorescence = citrullinated histone H3; red

568 fluorescence = MPO; NETs are highlighted by yellow arrows in the overlay images. $(F)$

569 Bar graph showing number of NETs crossing grid lines per high power field. One-way

570 ANOVA with Tukey's post hoc testing. ${ }^{* * *}$ denotes $p<0.001$. 
(105 CFU for Figure 1B

Streptomycin Oral gavage and $\mathrm{C}$ and $10^{10} \mathrm{CFU}$ for
the rest of the figures)

pretreatment S.Typhimurium $\left(10^{4} \mathrm{CFU}\right)$

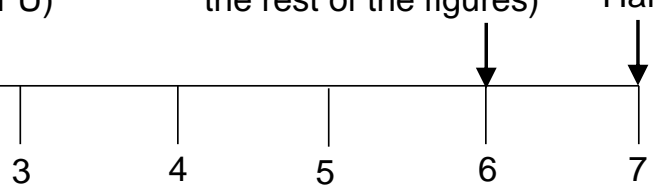

Days

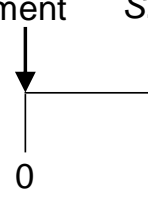

B

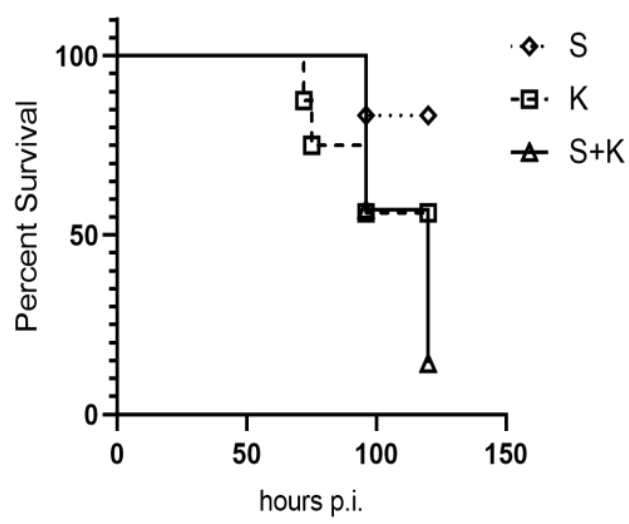

C

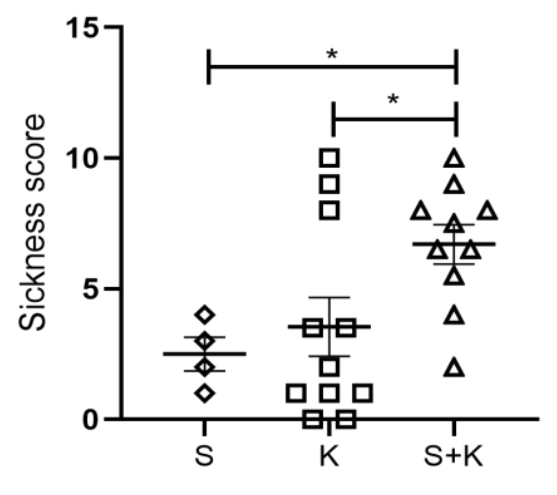

D

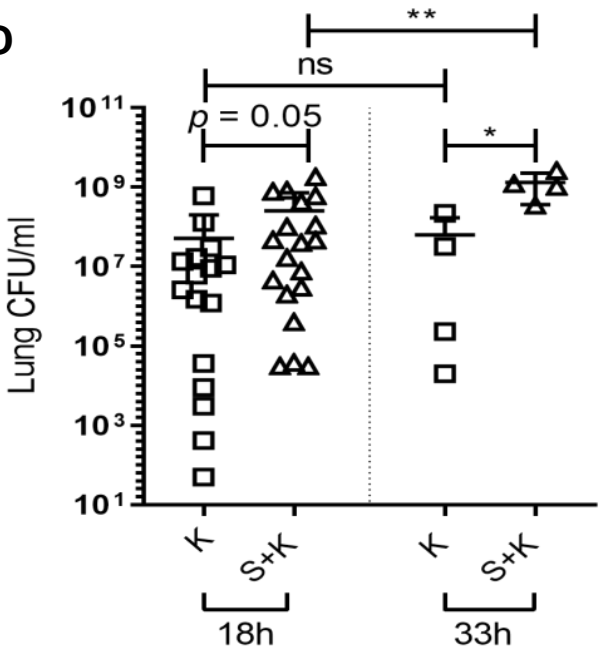


A Uninfected

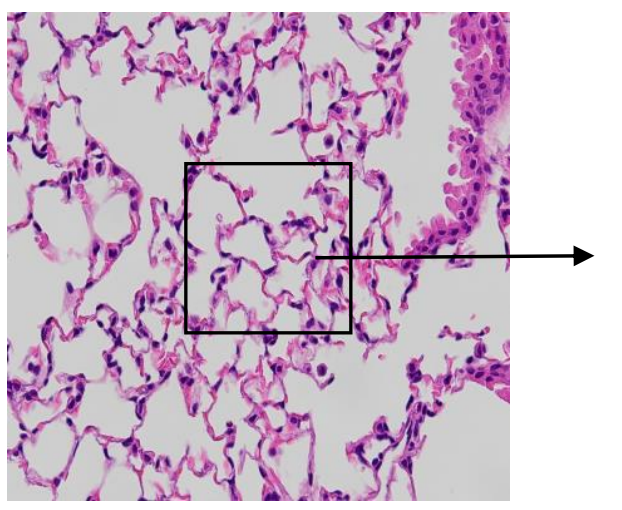

S

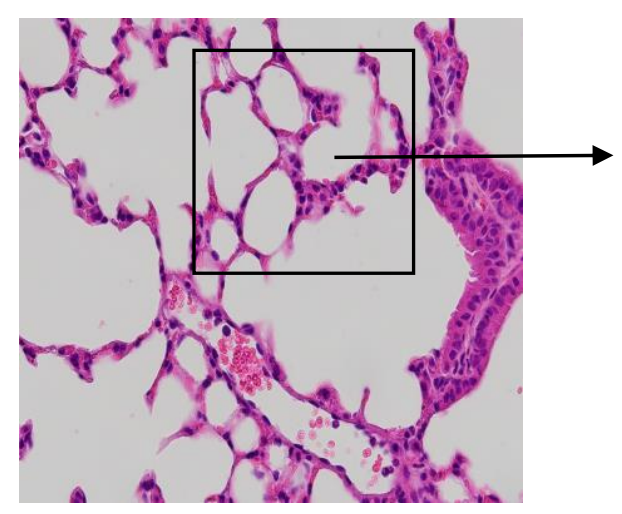

$\mathrm{K}$

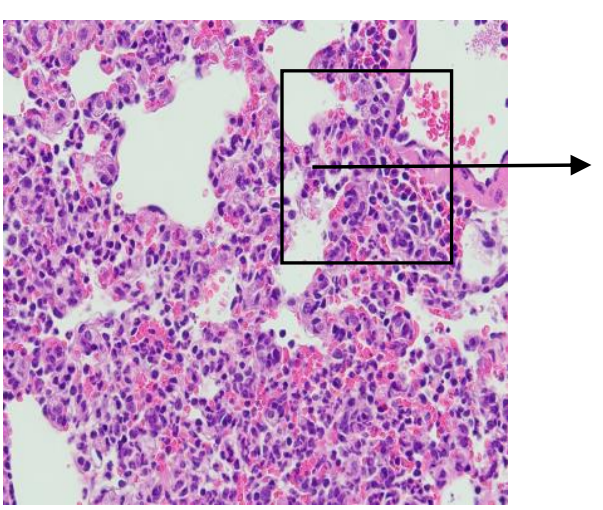

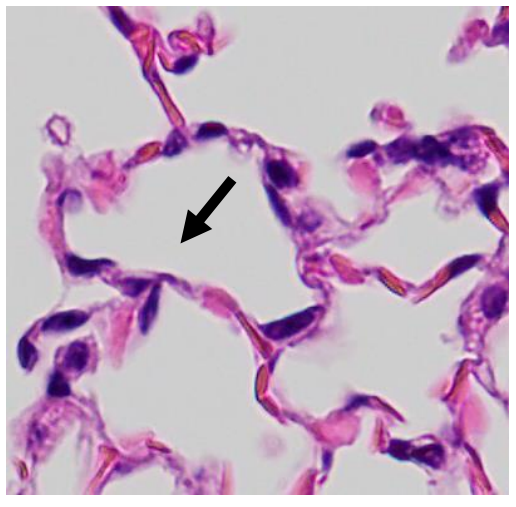

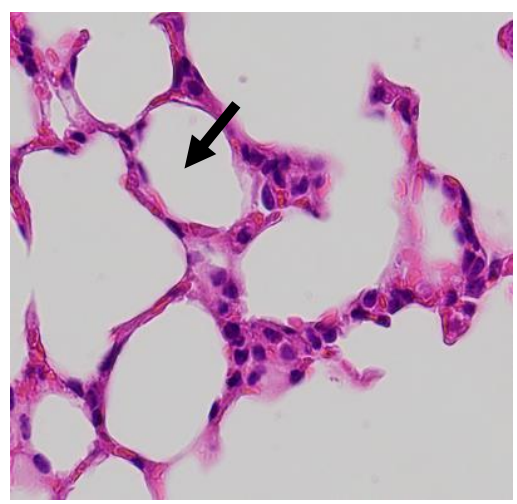

\section{$\mathrm{S}+\mathrm{K}$}

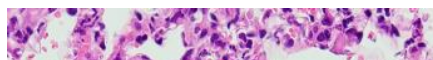

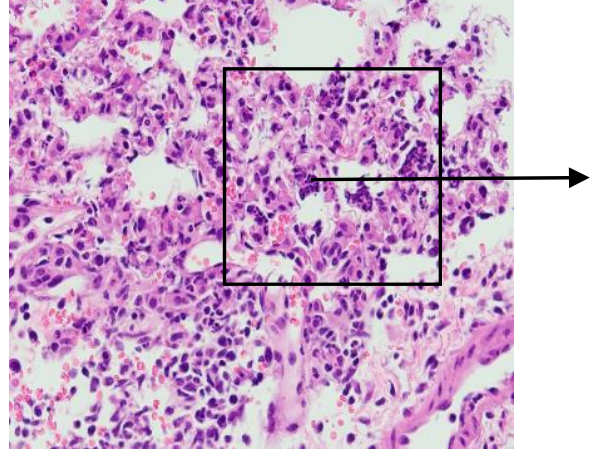

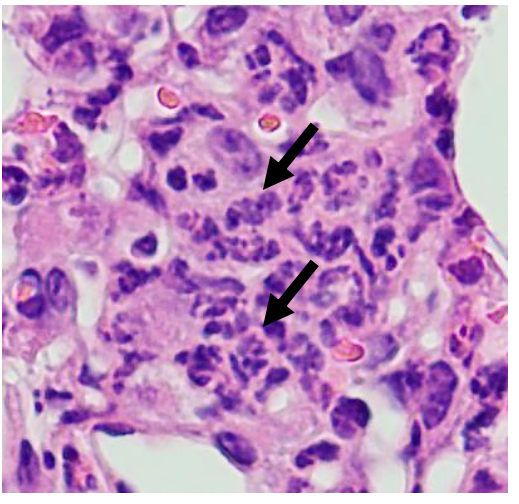

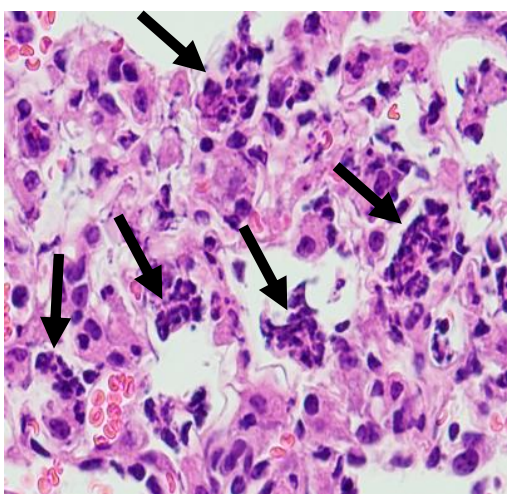

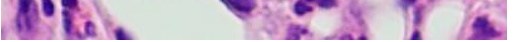

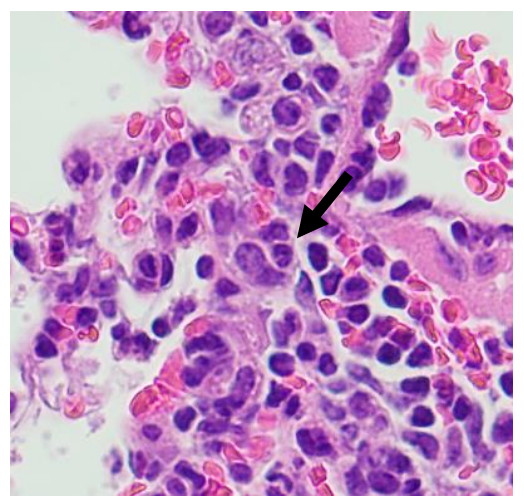

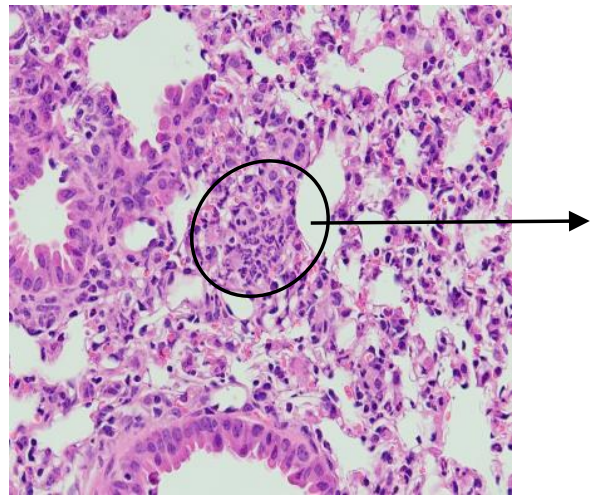




\section{B}
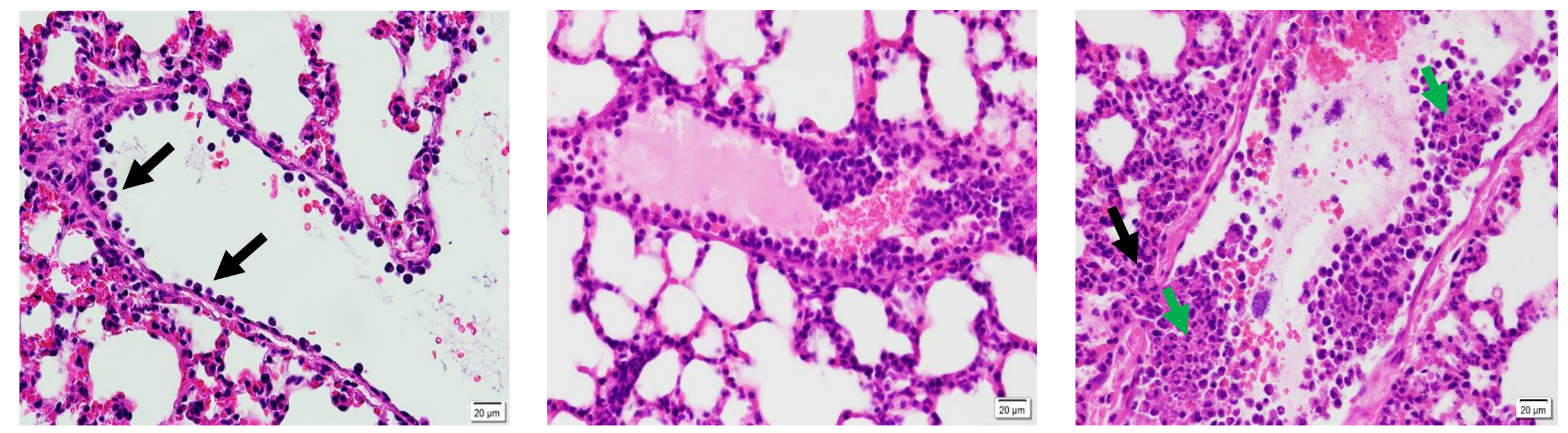

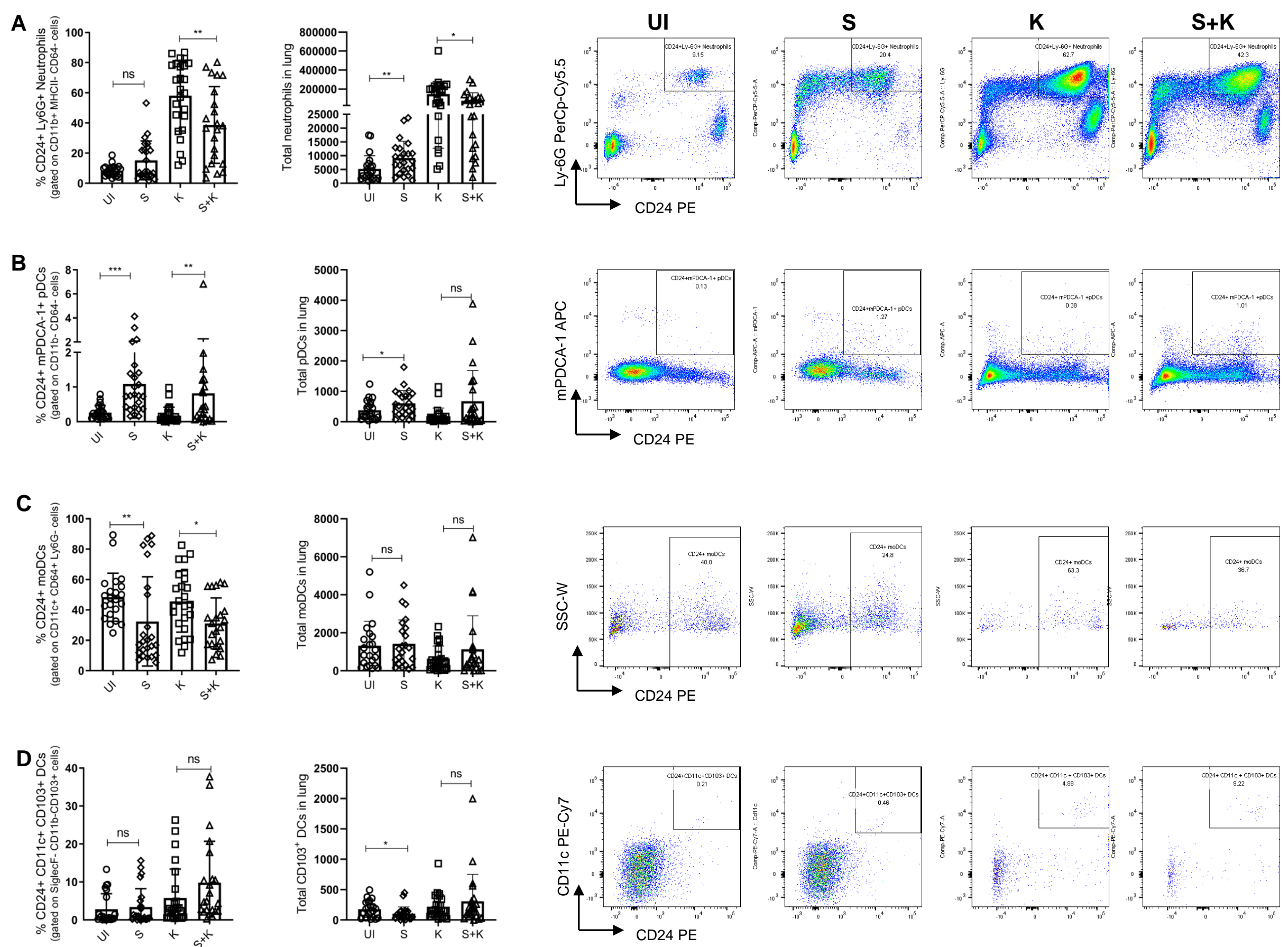
A

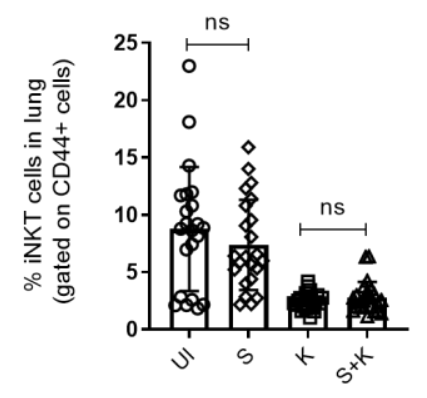

B

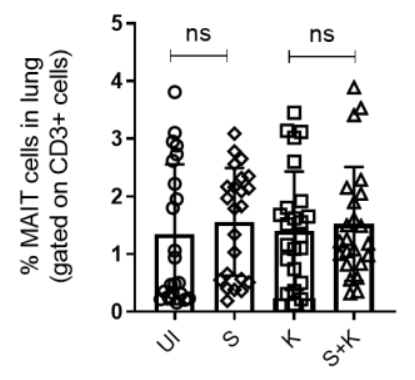

C

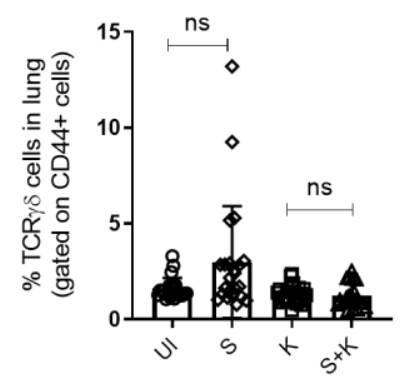

D

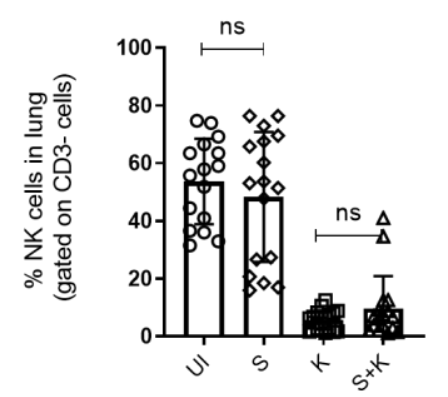

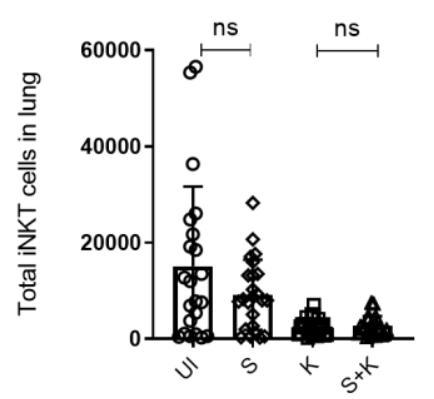
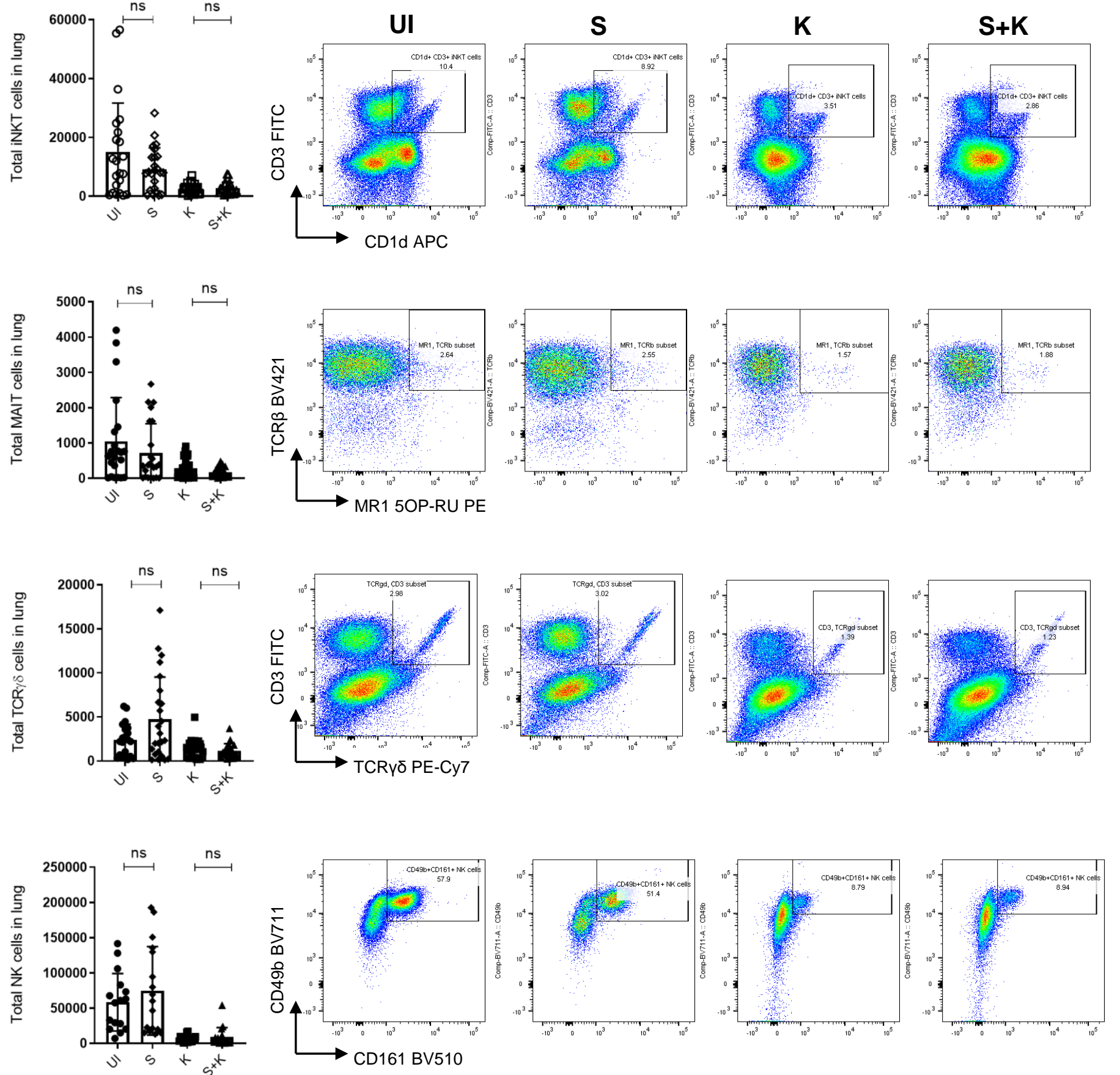


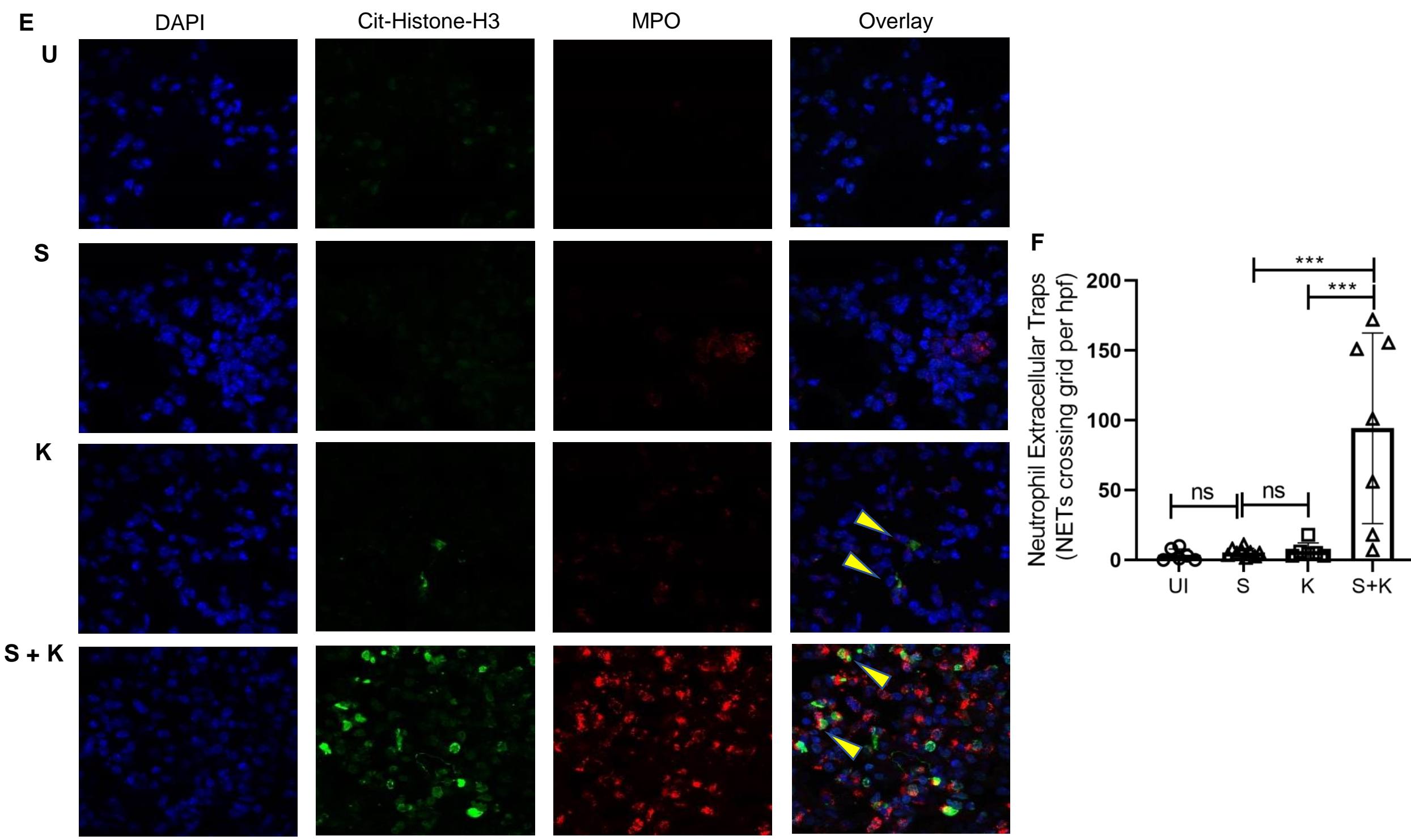

